\title{
Emergency Preparedness Training for Hospital Nursing Staff, New York City, 2012-2016
}

\author{
Jasmine L. Jacobs-Wingo, MPH ${ }^{1}$, Jeffrey Schlegelmilch, MPH, MBA², Maegan Berliner, $\mathrm{MPH}^{3}$, \\ Gloria Airall-Simon, PhD, MPHE, MSN, BSN ${ }^{4}$, \& William Lang, $\mathrm{MS}^{5}$
}

1 Temporary Epidemiology Field Assignee, Division of State and Local Readiness, Office of Public Health Preparedness and Response, Centers for Disease Control and Prevention, Atlanta, GA and Office of Emergency Preparedness and Response, New York City Department of Health and Mental Hygiene, New York, NY, USA

2 Deputy Director, National Center for Disaster Preparedness, Earth Institute, Columbia University, New York, NY, USA

3 Health and Medical Unit, New York City Emergency Management, Brooklyn, NY, USA

4 Senior Nurse Educator for Communicable Disease Preparedness, Office of Emergency Preparedness and Response, New York City, Department of Health and Mental Hygiene, New York, NY, USA

5 Director, Hospital Readiness and Health Care Coalitions, Office of Emergency Preparedness and Response, New York City Department of Health and Mental Hygiene, New York, NY, USA

Key words

Curriculum, nurses' disaster preparedness education

\section{Correspondence}

William Lang, 42-09 28th St. Long Island City, New York 11101

E-mail:wlang1@health.nyc.gov

Accepted June 6, 2018

doi:10.1111/jnu.12425

\begin{abstract}
Purpose: Many nurses are trained inadequately in emergency preparedness (EP), preventing them from effectively executing response roles during disasters, such as chemical, biological, radiological, nuclear, and explosive (CBRNE) events. Nurses also indicate lacking confidence in their abilities to perform EP activities. The purpose of this article is to describe the phased development of, and delivery strategies for, a CBRNE curriculum to enhance EP among nursing professionals. The New York City (NYC) Department of Health and Mental Hygiene (DOHMH) and the National Center for Disaster Preparedness at Columbia University's Earth Institute led the initiative.

Methods: Curriculum development included four phases. In Phases I and II, nursing staff at 20 participating NYC hospitals conducted 7,177 surveys and participated in 20 focus groups to identify training gaps in EP. In Phase III, investigators developed and later refined the CBRNE curriculum based on gaps identified. In Phase IV, 22 nurse educators (representing 7 of the original 20 participating hospitals) completed train-the-trainer sessions. Of these nurse educators, three were evaluated on their ability to train other nurses using the curriculum, which investigators finalized.

Findings: The CBRNE curriculum included six modules, a just-in-time training, and an online annual refresher course that addressed EP gaps identified in surveys and focus groups. Among the 11 nurses who were trained by three nurse educators during a pilot training, participant knowledge of CBRNE events and response roles increased from an average of $54 \%$ (range $45 \%-75 \%$ ) on the pre-test to $89 \%$ (range $80 \%-90 \%$ ) on the posttest.
\end{abstract}

Conclusions: By participating in nursing CBRNE training, nurses increased their knowledge of and preparedness to respond to disasters. The trainthe-trainer curriculum is easily adaptable to meet the needs of other healthcare settings.

Clinical Relevance: The CBRNE curriculum can be used to train nurses to better prepare for and more effectively respond to disasters. 
All across the world, manmade and natural catastrophic health events (CHEs) can cause inconceivable destruction to health and infrastructure. In New York City (NYC), CHEs including the September 11 terrorist attacks and Superstorm Sandy required enormous and wellcoordinated efforts from hospitals and response agencies (Klitzman \& Freudenberg, 2003; Office of Inspector General, 2015; VanDevanter, Kovner, Raveis, McCollum, \& Keller, 2014). Healthcare workers receiving and treating disaster victims must have clearly defined response roles and quality training to execute responses effectively (Gebbie \& Qureshi, 2006). Realizing the global need to strengthen capacities so nurses can carry out their pivotal roles during disasters, the International Council of Nurses (ICN) collaborated with the World Health Organization (2009) to develop competencies to describe nurses' response roles during disasters in the ICN Framework for Disaster Nursing Competencies. During in-servicing or clinical education, hospital healthcare providers receive emergency preparedness (EP) training limited in quality and frequency (Weiner, Irwin, Trangenstein, \& Gordon, 2005). Once in the workplace, many hospital healthcare providers receive EP training only once, infrequently, or never at all, thereby lessening the likelihood that staff will carry out their essential response roles during a $\mathrm{CHE}$ as intended.

Since 2002, the NYC Department of Health and Mental Hygiene (DOHMH) has received funding for healthcare emergency preparedness from various agencies within the U.S. Department of Health and Human Services (HHS). Such funds were largely distributed to hospitals via a Hospital Preparedness Program aimed at developing and maintaining acute care preparedness capabilities, initially for bioterrorism and later extending to all hazards (Office of the Assistant Secretary for Preparedness and Response, n.d.). With all of NYC's then 57 acute care hospitals needing funding, the DOHMH prioritized spending on hospitals most likely to engage in disaster response. Federal preparedness funds translated into grant projects and programs with work deliverables, many of which included staff training on hospital emergency department disaster preparedness and response activities.

In 2010, the HHS's Assistant Secretary for Preparedness and Response (ASPR) in collaboration with the Center for Biosecurity of University of Pittsburgh Medical Center (2009) published The Next Challenge in Healthcare Preparedness: Catastrophic Health Events. The report postulated that the U.S. healthcare system is unprepared for catastrophic disasters (e.g., a severe pandemic or nuclear detonation) and recommended that all U.S. hospitals "participate in a healthcare coalition that prepares and responds collaboratively to common medical disasters and CHEs" (p.24).
The ASPR challenged emergency managers (funders and providers alike) to think more broadly about their preparedness program needs by integrating the concept of "response" in planning considerations.

By 2012, the DOHMH began introducing training, protocols that allowed hospitals to expand bed surge capacity through rapid patient discharge, and exercises that encouraged hospitals to look for ways of incorporating disaster preparedness in their inpatient operations. These various initiatives revealed a major gap: during CHEs or other scenarios that overwhelm the emergency department, hospital floor nurses from multiple service areas are unprepared to execute their response roles and receive disaster victims arriving directly on their units. The DOHMH's Bureau of Healthcare System Readiness consulted with the National Center for Disaster Preparedness at Columbia University's Earth Institute to research and close this apparent gap, the result of which was an emergency preparedness curriculum for nurses that encompasses chemical, biological, radiological, nuclear, and explosive (CBRNE) events. The purpose of this article is to describe the phased development of delivery strategies for a CBRNE curriculum to enhance EP among nursing professionals.

Nurses (particularly those in midlevel management positions and the staff they supervise) were the target audience for this new curriculum because they represent a major proportion of the healthcare workforce. According to U.S. Bureau of Labor Statistics (2016) data, nurses represented one of the largest occupational groups in both the "outpatient care center" and the "general medical and surgical hospitals" industries. Registered nurses, in particular, represented $15.4 \%$ and $30.5 \%$ of total employment in these industries, respectively. Nurses were also targeted because their response roles during disasters have a substantial impact on the hospital level or hospital unit level. For example, during Hurricane Irene and Superstorm Sandy, nurses were heavily involved in patient evacuation procedures. Nurses at sending facilities organized unit-based activities to facilitate the transport of remaining patients to other facilities, and nurses at receiving facilities rapidly discharged patients and made other preparations to receive incoming patients (VanDevanter, Raveis, Kovner, McCollum, \& Keller, 2017). Furthermore, disasters could require nurses to work in unfamiliar units, highlighting the importance of EP training.

\section{Methods}

\section{Determining Emergency Preparedness Gaps Among Nurses}

The nursing CBRNE training project was divided into four development phases (Figure 1). Twenty hospitals 


\section{REFINEMENT \& IMPLEMENTATION: Phase IV (2015-2016)}

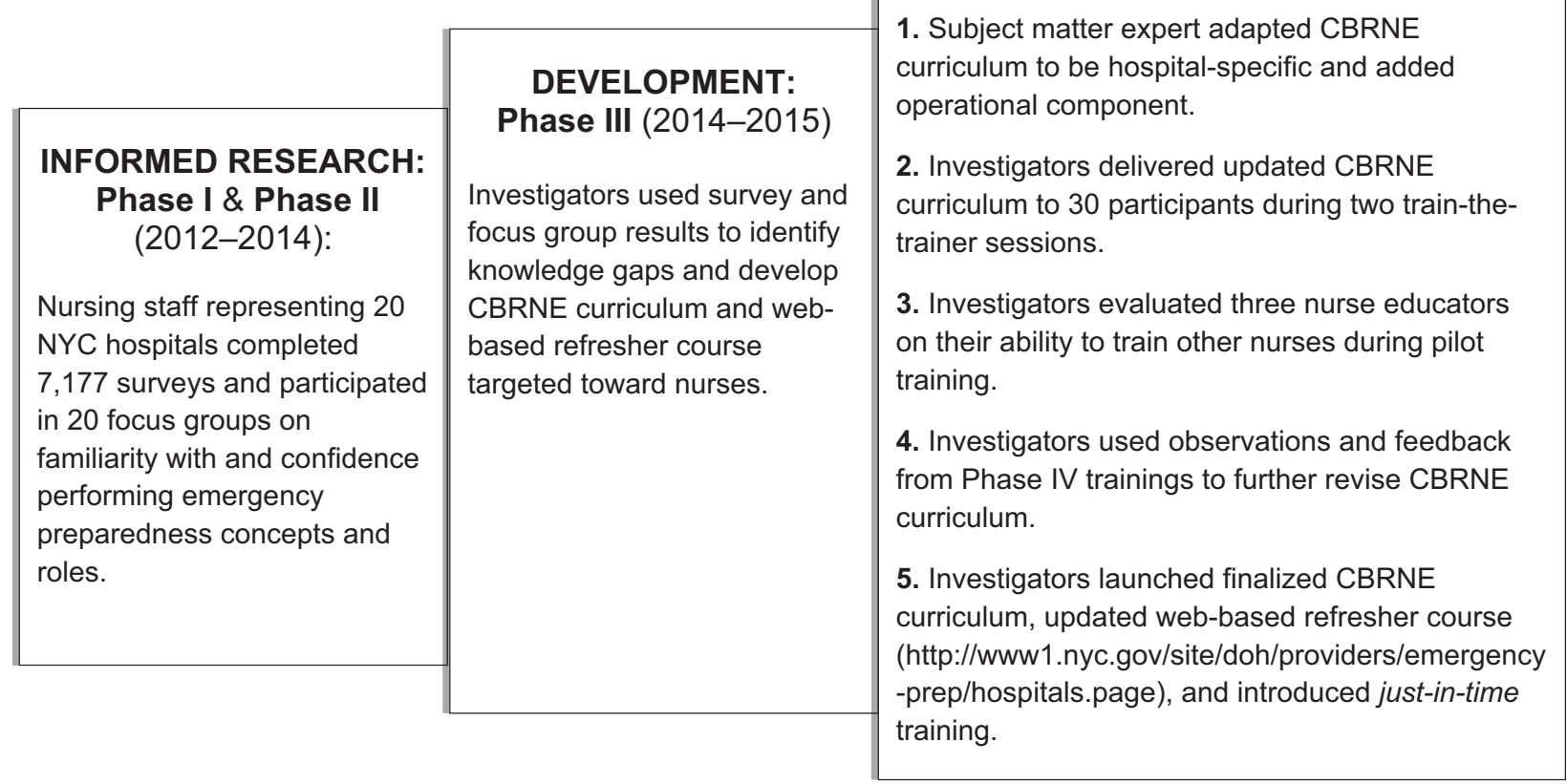

Figure 1. Phased process of chemical, biological, radiological, nuclear, and explosive (CBRNE) curriculum development for training of hospital nursing staff.

within the five boroughs of NYC participated in Phase I and Phase II of the project, which occurred from 2012 through 2013. During these initial phases, investigators conducted 20 separate hour-long focus groups (one at each participating hospital) to capture nursing staff's personal experiences and perceived EP gaps. Investigators then used focus group findings to develop an online survey. Survey items were reviewed by subject matter experts at the DOHMH and National Center for Disaster Preparedness for content validity. The survey was pilot tested in a convenience sample of nurses and hospital staff. Namely, the survey assessed familiarity with EP concepts and confidence (i.e., self-reported assessment of one's comfort or ability) performing EP job duties during various scenarios. More than $36 \%$ of nursing staff $(N=7,177)$ at participating hospitals completed the survey. The majority of respondents worked full time $(87.2 \%)$, were registered nurses $(74.2 \%)$, and maintained nonsupervisory positions (75.8\%). Respondents indicated having worked in a hospital setting for an average of 16.7 years (range 0 to 75 years).

\section{Developing First Iteration of Curriculum}

During Phase III (2014-2015), investigators developed a nurse-specific CBRNE train-the-trainer curriculum and accompanying facilitator guide and participant manual. The curriculum addressed major gaps identified during the preceding phases. Investigators chose to train nurse educators on EP concepts-a train-thetrainer approach-rather than training the nurses themselves because of sustainability and potential for wider reach. Investigators solicited feedback on the CBRNE train-the-trainer curriculum during two additional focus groups with 14 nurses (with and without prior EP experience or training). The Phase III curriculum also incorporated an EP-focused, less time intensive, online annual refresher training program to ensure participants retained knowledge from the initial training.

\section{Refining, Implementing, and Evaluating Program Curriculum}

Early during Phase IV of the project (2015-2016), an external hospital preparedness coordinator with a nursing background updated the Phase III curriculum by adding more hospital-specific content (e.g., integrating the hospital's incident command system [ICS]) and removing content that exceeded the scope of nursing practice.

Nurse educators were recruited from a convenience sample by sending an e-mail describing objectives of 
the project, training benefits, and staff participation requirements to nurse educators at seven facilities that participated in prior project phases. Using the revised curriculum, investigators delivered two train-the-trainer sessions (hosted at NYC hospitals) in the spring and summer of 2015 for nurse educators.

In June 2015, investigators evaluated a selection of nurse educators who had attended the CBRNE training on their ability to deliver the curriculum during a pilot training. Nurse educators were evaluated using concepts from the Kirkpatrick model-a method for evaluating training programs based on trainees' reaction to the program, understanding of program knowledge or skills, ability to perform, and impact of training (Kirkpatrick, 1996; Smidt, Balandin, Sigafoos, \& Reed, 2009; Speakman, 2017). To further evaluate the pilot training, investigators developed a web-based platform that nurse educators used to register their classes and on which students of these classes submitted answers to pre- and posttests.

In Phase IV, investigators incorporated the preferred training formats of those surveyed in Phase I and Phase II: just-in-time, train-the-trainer, and online. Investigators used feedback from training attendees and observations from both the initial train-the-trainer sessions and pilot training to further revise the nurse educator curriculum, to create an online annual refresher training (lasting 45-60 $\mathrm{min}$ ) that reviews concepts presented in the instructor-led training, and to create a just-in-time training module (lasting 5-10 min) that can rapidly provide training to hospital nursing staff on how to respond to CBRNE events.

This study was determined to be a public health activity not involving research with human subjects (HSR\#17062301); therefore, no formal institutional review board approvals were required. The Paperwork Reduction Act did not apply.

\section{Results}

The survey assessed previous EP training. Of nurses who responded on their training, areas in which most had not been trained included preparedness for radiological and nuclear agents $(82.6 \%)$, preparedness for biological agents $(79.4 \%)$, preparedness for chemical agents $(74.6 \%)$, hazardous materials and patient decontamination $(73.5 \%)$, and their own role within the hospital's ICS (62.5\%). Patient evacuation (59.9\%) and the hospital's EP plan (53.5\%) were the only areas in which most respondents had been trained. The survey also assessed respondents' confidence performing EP activities. The greatest number of respondents $(n=7,174)$ reported lacking confidence in treating patients exposed to a radioactive material (78.3\%), treating patients exposed to a biological agent $(76.1 \%)$, and performing decontamination procedures (73.7\%). Evacuating patients from units, departments, or hospitals was the only activity in which more respondents $(59.9 \%)$ reported having confidence than lacking confidence. When respondents $(n=7,176)$ were asked about their incentives for participating in a "nonrequired" training program, the top incentives were having no costs to complete the training $(83.1 \%)$ and receiving continuing education credits $(79.2 \%)$.

The 4-hr finalized CBRNE curriculum is divided into six modules with detailed footnotes and a pre- and posttest. Overall, the training introduces CBRNE agents and events, their relevance to everyday life, and describes the roles of nurses in responding to such events. Modules include Hospital Disaster Response and hospital-based nurse readiness for Chemical Agents, Biological Agents, Radiological/Nuclear Agents, Hospital Decontamination, and Explosives and Blasts (Figure 2).

Thirty individuals (22 in the first session and 8 in the second session) representing seven hospitals of differing sizes, networks, or specialties completed one of two train-the-trainer sessions in Phase IV. Nonnursing staff included seven paramedics, two emergency managers, one radiation safety officer, and one planning director. Of 27 individuals completing post-session evaluations, $100 \%(n=27)$ agreed or strongly agreed that the CBRNE training enhanced their knowledge of the subject matter and was relevant for what they might be expected to do in an emergency, and $81.5 \%$ $(n=22)$ indicated that the training contributed to their ability to lead or manage others in an emergency to a great extent.

Of those who received CBRNE training, three nurse educators were evaluated on their ability to deliver the curriculum during a pilot training housed at an NYC hospital and attended by 11 nurses. During the pilot training facilitated by nurse educators, scores of training attendees increased from an average of $54 \%$ (range $45 \%-75 \%$ ) on the pre-test to $89 \%$ (range $80 \%-90 \%)$ on the posttest.

\section{Discussion}

Deliberate attacks present a major threat to public safety; hospitals and other healthcare facilities should increase their emergency preparedness for these threats among nurses and other staff. During an iterative process, investigators gleaned feedback from nursing staff throughout focus groups and thousands of surveys to develop a curriculum with the goal of increasing nurses' preparedness for CBRNE events. Surveys and focus 

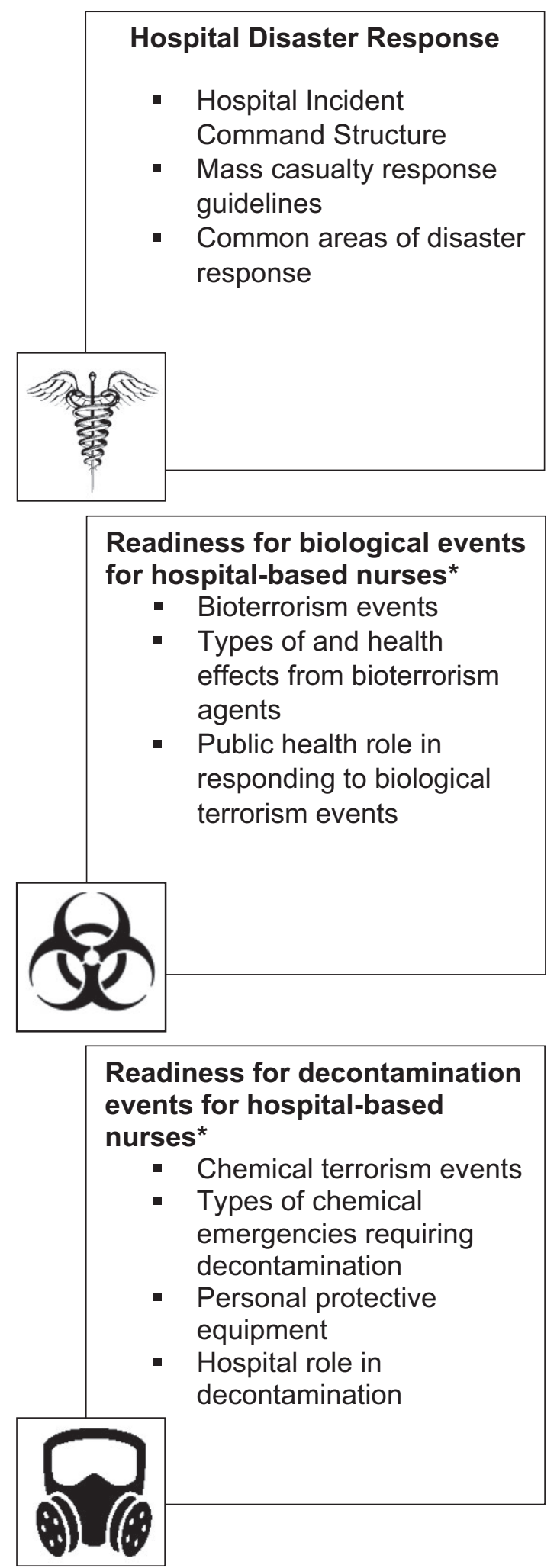
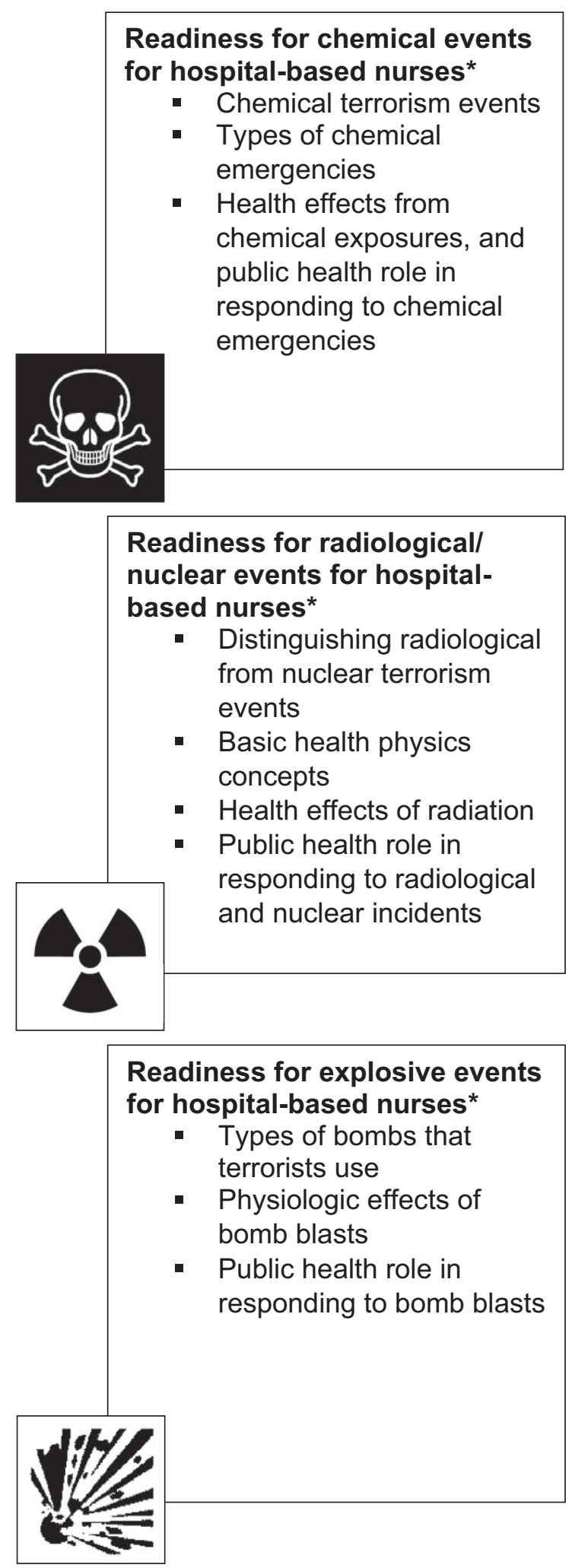

*Module also included in Phase III curriculum.

Figure 2. Modules included in Phase IV of the chemical, biological, radiological, nuclear, and explosive (CBRNE) curriculum for nursing staff emergency preparedness training. 
groups confirmed investigators' presumptions and other research findings; overall, nursing staff lacks confidence in their ability to perform EP activities (Baack \& Alfred, 2013). As demonstrated during the train-the-trainer sessions and pilot training, the CBRNE curriculum successfully contributed to the ability to lead or manage others in an emergency and increased knowledge of emergency preparedness among NYC nurses. We presume the curriculum also better prepared participants to perform their response functions in the context of CBRNE events, but the program's impact on actual performance during disasters has not been examined. Our small sample size limits the generalizability of results. Given the standardized role of hospitals and clinical presentations from CBRNE exposure, however, we believe the curriculum would have the same effect with a larger group of nursing staff or one outside NYC.

Investigators have considered ways to increase the number of nurses completing the CBRNE curriculum. The curriculum contains the top incentives that respondents identified. For example, the CBRNE curriculum is free and, by design, already meets many continuing education requirements for training programs such as including pre- and posttests that measure knowledge change and the attainment of prespecified objectives. Others should explore mechanisms for offering formal continuing education credits when implementing the CBRNE curriculum.

As an additional tactic to increase participation, hospitals could explore presenting CBRNE modules gradually, rather than all at once during a multihour session. Hospitals could integrate the curriculum into monthly professional development activities, such as "grand rounds," so training does not become an additional obligation. Other jurisdictions adapting the curriculum for their own EP training needs may want to consider these and other incentives to maximize the number of participants trained.

When implementing the CBRNE curriculum, healthcare facilities may also consider using multiple instructors. Nurse educators who facilitated the train-the-trainer pilot sessions indicated that they were not subject matter experts in all concepts covered by the CBRNE curriculum, but could apply expertise to enhance certain modules. If possible, using multiple instructors to present the CBRNE curriculum would lessen the burden on any one individual to present.

An important aspect of the CBRNE curriculum is its adaptability to multiple healthcare settings and professions. While the curriculum was developed for a nursing audience, other professionals (e.g., paramedics) also attended train-the-trainer sessions, suggesting interest among other professions. The DOHMH has released the CBRNE curriculum in an editable format so that healthcare facilities can adapt it to their own needs, including the needs of various healthcare professionals like nonhospital nurses. Similarly, the online refresher training (as made available upon request to the DOHMH) can be hosted on any Learning Management System, making the training more accessible.

The risk for CBRNE events and other disasters will vary by jurisdiction. NYC, like other major metropolises, has been targeted for deliberate attacks. Other jurisdictions, large or small, all across the globe, should also prepare to respond to catastrophic events. We encourage readers to access the DOHMH's emergency preparedness webpage (see Figure 1) and utilize this free, publicly available resource in their own jurisdictions to increase the resiliency of healthcare facilities to respond to disasters.

\section{Acknowledgments}

This report was supported by Cooperative Agreement Numbers 1U90TP000546-01, 5U90TP000546-02, and 5U90TP000546-03 funded by the Centers for Disease Control and Prevention. Its contents are solely the responsibility of the authors and do not necessarily represent the official views of the Centers for Disease Control and Prevention or the Department of Health and Human Services. The authors would like to acknowledge Elaine Vernetti, MD, MPH, for her thorough review of and detailed revisions to the nursing curriculum.

\section{Clinical Resource}

- NYC Health. Hospitals, emergency preparedness. http://wwwl.nyc.gov/site/doh/providers / emergency-prep/hospitals.page

\section{References}

Baack, S., \& Alfred, D. (2013). Nurses' preparedness and perceived competence in managing disasters. Journal of Nursing Scholarship, 45(3), 281-287. https://doi.org/10.1111/jnu.12029

Bureau of Labor Statistics, U.S. Department of Labor. (2016). Data tables for OES industry charts. Retrieved from https://www.bls.gov/oes/current/ind_emp_chart/ ind_emp_chart_data.htm\#62

Center for Biosecurity of University of Pittsburgh Medical Center. (2009). The next challenge in healthcare preparedness: Catastrophic health events. 
Baltimore, MD: U.S. Department of Health and Human Services.

Gebbie, K., \& Qureshi, K. (2006). A historical challenge: Nurses and emergencies. Online Journal of Issues in Nursing, 11(3), 2.

Kirkpatrick, D. (1996). Great ideas revisited. Techniques for evaluating training programs. Revisiting Kirkpatrick's four-level model. Training and Development, 50, 54-59.

Klitzman, S., \& Freudenberg, N. (2003). Implications of the World Trade Center attack for the public health and health care infrastructures. American Journal of Public Health, 93(3), 400-406.

Office of the Assistant Secretary for Preparedness and Response, U.S. Department of Health and Human Services. (n.d.). Hospital Preparedness Program. Retrieved from http://www.phe.gov/preparedness/ planning/hpp/pages/default.aspx

Office of Inspector General, U.S. Department of Health and Human Services. (2015). Hospital emergency preparedness and response during Superstorm Sandy. Journal of Healthcare Protection Management, 31(1), 31-50. Retrieved from https:// oig.hhs.gov/oei/reports/oei-06-13-00260.pdf

Smidt, A., Balandin, S., Sigafoos, J., \& Reed, V. A. (2009). The Kirkpatrick model: A useful tool for evaluating training outcomes. Journal of Intellectual and Developmental Disability, 34(3), 266-274. https:// doi.org/10.1080/13668250903093125

Speakman, E. (2017). Interprofessional education and collaborative practice: Creating a blueprint for nurse educators. Philadelphia, PA: National League for Nursing; Wolters Kluwer.

VanDevanter, N., Kovner, C. T., Raveis, V. H., McCollum, M., \& Keller, R. (2014). Challenges of nurses' deployment to other New York City hospitals in the aftermath of Hurricane Sandy. Journal of Urban Health, 91(4), 603-614. https://doi. org/10.1007/s1 1524-014-9889-0

VanDevanter, N., Raveis, V. H., Kovner, C. T., McCollum, M., \& Keller, R. (2017). Challenges and resources for nurses participating in a Hurricane Sandy hospital evacuation. Journal of Nursing Scholarship, 49(6), 635-643. https://doi.org/10.1111/jnu.12329

Weiner, E., Irwin, M., Trangenstein, P., \& Gordon, J. (2005). Emergency preparedness curriculum in nursing schools in the United States. Nursing Education Perspectives, 26(6), 334-339.

World Health Organization $\&$ International Council of Nurses. (2009). ICN framework of disaster nursing competencies. Geneva, Switzerland: Author. Retrieved from http://www.icn.ch/images/stories/documents/ networks/DisasterPreparednessNetwork/Disaster_ Nursing_Competencies_lite.pdf 